\title{
Temporomandibular joint dislocation: a retrospective study from a Swiss urban emergency department
}

This article was published in the following Dove Press journal: Open Access Emergency Medicine

\author{
Giorgos Papoutsis' \\ Sylvana Papoutsi \\ Jolanta Klukowska-Rötzler \\ Benoît Schaller ${ }^{3}$ \\ Aristomenis K Exadaktylos \\ 'Department of Emergency Medicine, \\ ${ }^{2}$ Department of Visceral Surgery and \\ Medicine, ${ }^{3}$ Department of Cranio- \\ Maxillofacial Surgery, Inselspital, \\ University Hospital Bern, University \\ of Bern, Bern, Switzerland
}

Correspondence: Jolanta

Klukowska-Rötzler

Department of Emergency Medicine, University Hospital Bern, Inselspital, University of Bern, Freiburgerstrasse 16C, 30I0, Bern, Switzerland

$\mathrm{Tel}+4|3| 6323396$

Email jolanta.klukowska-roetzler@insel. ch
Purpose: Temporomandibular joint (TMJ) dislocation is an uncommon and debilitating condition of the facial skeleton. The condition may be traumatic or nontraumatic, in an acute or chronic form, and with bilateral or monolateral expression.

Patients and methods: In this study, conducted from May 2012 to July 2016, we retrospectively analyzed TMJ dislocations treated in the Department of Emergency Medicine, Inselspital, University Hospital Bern, University of Bern, by focusing on the following parameters: age, gender, reason, localization, frequency, and therapy.

Results: Thirty-two patients were included. The mean age was 42.06 years and there was no predominant gender. Most cases of TMJ dislocation were nontraumatic (93.7\%). Dislocations were mostly bilateral (59.4\%) and appeared in a chronic situation and with repetitive events (62.5\%). Thirty-one patients received conservative treatment, which consists of reposition of the TMJ with $(38.7 \%)$ or without $(61.3 \%)$ analgosedation. Only one patient needed surgical reposition due to previous surgical treatment.

Conclusion: This is the first attempt to evaluate TMJ dislocations in Switzerland in an acute hospital setting. To our knowledge, there are no other studies that systematically analyze these injuries by focusing on the patients' characteristics. Surgical reposition is only indicated in complicated and very rare situations. Conservative approaches are commonly used and should be exhausted before any surgery.

Keywords: temporomandibular joint, emergency medicine, dislocations

\section{Introduction}

The temporomandibular joint (TMJ) is a joint between the mandible and the temporal bone of the skull. The condyle of the mandible articulates bilaterally in a concavity known as the glenoid fossa or mandibular fossa. The movements of the TMJ are under neuromuscular control and comprise the muscles of mastication, the ligaments associated with the TMJ, and neural transmission carried by the mandibular division of the trigeminal nerve. ${ }^{1,2}$ TMJ dislocation occurs when one or both mandibular condyles are displaced in front and above the articular eminence. Dislocation may be reducible if the condyle returns spontaneously to the glenoid cavity (subluxation) or irreducible when one or two condyles remain dislocated (luxation). In the latter condition, the mouth remains open and the front teeth do not meet, due to the action of the elevator muscles with or without lateral deviation, and depending on whether the dislocation is unilateral or bilateral. ${ }^{3}$ Dislocation of the joint may be uni- or bilateral, anterior, posterior, superior, or lateral. Anterior dislocations are by far the most common. 
The other dislocation types are commonly associated with trauma and concurrent fractures. ${ }^{4}$ Further classification of TMJ dislocations includes the associated causes, which are spontaneous, iatrogenic, or traumatic.

The etiopathogenesis of spontaneous dislocation of the $\mathrm{TMJ}$ is generally unknown. It frequently happens in relation with yawning and less often after mild facial trauma (such as a slap to the chin) or in the setting of laughing. ${ }^{5,6}$ Several theories have been put forward to explain the onset of TMJ dislocation. It is commonly associated with poor development of the articular fossa, laxity of the temporomandibular ligament or joint capsule, and excessive activity of the lateral pterygoid and infrahyoid muscles due to drugs, such as phenothiazines, metoclopramide, or systemic diseases (Parkinson's, multiple sclerosis, and epilepsy). ${ }^{6-8}$ It may also be related to some neuromuscular disorders or disorders of collagen metabolism, such as ligamentous hyperlaxity, Ehlers-Danlos syndrome, or Duchenne muscular dystrophy, which cause a predilection for joint laxity. ${ }^{6,7,28}$ Furthermore, overclosure in edentulous patients is considered as a factor influencing the condyle position. The TMJ dislocation has an incidence of about $3 \%$ of all dislocations throughout the body. ${ }^{9}$ Spontaneous anterior TMJ dislocation is not a common condition, with a reported annual incidence of 5.3 per 1,000,000 patients who present to the emergency department (ED). ${ }^{10}$ The literature reports female predominance and, according to a study, the condition seems to be linked to hormonal imbalance. ${ }^{5}$ The most common TMJ dislocation is the anterior subtype. ${ }^{11,12}$ Posterior, superior, or lateral dislocations of an intact condyle are very rare; this rarity may be attributed to differences in the anatomy of the condyle, the direction of pull of muscles attached to the condyle, and the low incidence of skull base fractures from an indirect blow. ${ }^{11}$ Although the incidence is relatively low, it is crucial to address TMJ dislocation, as it has a vital and immediate impact on secretions needed for airway patency, as well as on pain. TMJ dislocation also has long-term effects on mastication and clarity of speech. ${ }^{13}$

The diagnosis of TMJ dislocation is based on clinical characteristics. After initial inspection, a functional examination is performed, which consists of both active movements - active closing of the mouth, active deviation of the mandible, and active forward protrusion of the chin - and resisted movements - resisted opening of the mouth, resisted deviation of the mandible, and resisted forward protrusion of the chin. Finally, the joint and masticatory muscles are palpated and the mandibular fossa is evaluated for emptiness in which the bony structure of the condyle is not palpated. To evaluate the integrity of the $\mathrm{TMJ}$, a radiological imaging such as the orthopantomogram, cone beam, or 3D computed tomography can be performed. MRI is not commonly used to evaluate TMJ dislocation, but is indicated to evaluate chronic TMJ changes like degenerative processes, disc dislocations, or lesions of the discs.

TMJ dislocation is classified as acute, chronic, or chronic recurrent. ${ }^{14}$ Chronic dislocations are described as acute dislocations that are not self-limiting and that progress without treatment.

TMJ dislocation can be reduced with a conservative approach, with or without analgosedation. Manual reduction is mostly chosen and can be accomplished by several methods, such as the Nélaton maneuver, the Hippocratic method, the wrist pivot, the extraoral method, and the combined ipsilateral method. The Hippocratic method is the most frequently described; the examiner stands in front of the patient and places a gloved thumb on the posterior lower molars bilaterally with fingers wrapped laterally around the mandible. ${ }^{7}$ Further conservative treatments, such as the use of an occlusal splint ${ }^{15,16}$ or TMJ extraoral automobilization are used in chronic dislocation. Sclerosing agents (alcohol, rivanol, 5\% sodium psylliate, autologous blood injection [ABI], and botulinum A toxin) have been injected into the joint cavity in patients with recurrent $\mathrm{TMJ}^{17}$ dislocations, but are no longer used due to the side effects. ABI and prolotherapy can be considered in case of recurrent dislocation. Periodic exercise programs ("Rocabado's 666 exercise protocol") ${ }^{18}$ are used as a conservative treatment in some cases of recurrent TMJ dislocation. ${ }^{19}$

\section{Surgical approach}

If the conservative treatment is not effective and there are alterations to the TMJ ligaments, musculature, and bony components, surgery may be necessary. ${ }^{7}$ This can be performed in the following cases:

1. In patients with ligament alterations, a capsular plication or ligamentorrhaphy can be performed. ${ }^{11,20}$

2. In patients with muscular alterations, the ligation of the coronoid to the zygomatic arch or scarification of temporalis tendon/temporalis myotomy can be done. ${ }^{11}$

3. In patients with bony alterations, the possible surgical techniques include bone hook, condylectomy, ramus osteotomies, eminoplasty, or total joint replacement. ${ }^{20,21}$

\section{Patients and methods}

This retrospective observational study over 4 years and 2 months (May 2012 to July 2016) comprises adult patients 
( $\geq 16$ years old) admitted to our ED after an accident or sudden event, which caused TMJ injury symptoms. All patients presenting in the ED with a TMJ dislocation during the study period were included in our study. Patients were identified using a search string in the patient demographic field of our computerized patient database (E.Care, Qualicare; Qualidoc AG., Trimbach, Switzerland). This medical database allows instantaneous recall of past diagnostic reports, consultations, X-rays, and other relevant medical documents. These databases were used to identify all patients presenting with temporomandibular joint. Cases were retrieved using a comprehensive full-text search algorithm with temporomandibular joint and related terms (TMJ, temporomandibular joint dislocation, TMJ dislocation), including their German translation, as search terms (Kieferluxation, Kiefersperre, Kiefersubluxation, Luxation Temporomandibular - Gelenk, temporomandibular, Kiefergelenk, and Kieferköpfchen). The data were fully anonymized before analysis and therefore, according to local law, no prior informed consent or approval by the ethics committee was necessary. Furthermore, each patient is obligated to sign a form in which he or she declares that the patient data can be used in an anonymized way for research purposes. Initially, 67 patients were found with the mentioned search string in the stated time period. After evaluation of each case, 35 patients were excluded because the TMJ was listed as a past event in the section of the secondary diagnosis. In the end, 32 patients were included in the study.

Comorbidities were taken into consideration if they altered the anatomy of the TMJ or had an effect on the movements of the TMJ. The inclusion criteria for comorbidities were connective tissue disease, eg, Ehlers-Danlos syndrome, torn ligaments, recurrent dislocations, anatomic changes of the fossa and articular eminence, weakness of the capsule of temporomandibular ligaments, and anatomical changes due to previous operations.

Statistical evaluation was purely descriptive, due to the small sample sizes. On this basis, a retrospective analysis of the patients with TMJ joint dislocation was performed, including gender, age, etiology (spontaneous or traumatic), side (right, left, and bilateral), whether this was a recurrence or a first-time event, the treatment method, which includes conservative approaches (with or without analgosedation) or surgery, as well as any related comorbidities. The treatment was chosen individually for each patient by the attending maxillofacial surgeon on call after evaluation of the above criteria.

\section{Results}

During the studied period, from May 2012 to July 2016, a total of $32(n=32)$ cases with TMJ dislocations were reported in our ED. Fifty percent $(n=16)$ of all cases reported were male. A total of $19(59.4 \%)$ patients with TMJ dislocation were under the age of 40 years. The median age was 42.06 years.

The detailed characteristics of each patient are shown in Table 1.

Additionally, 93.7\% $(n=30)$ of incidents were nontraumatic and the TMJ dislocation then occurred triggered by one of the following circumstances: during eating $(n=2)$, after an epileptic seizure $(n=2)$, during an acute psychosis event $(n=1)$, while vomiting $(n=1)$, or during dental treatment $(n=1)$. No trigger was given for the 23 remaining patients with spontaneous dislocation (76.7\%). Only $6.3 \%(n=2)$ of the patients had a traumatic TMJ dislocation, and in both cases this was a result of a fall.

Nineteen $(59.4 \%)$ patients suffered bilateral dislocation, 10 patients $(31.2 \%)$ left-sided dislocation, and only three patients $(9.4 \%)$ right-sided TMJ dislocation (Figure 1). For 20 patients $(62.5 \%)$, this was a chronic situation with recurrent events and 12 patients $(37.5 \%)$ referred to the TMJ dislocation as being a first-time event.

Thirty-one patients $(96.9 \%)$ were given conservative treatment and 19 of these $(61.3 \%)$ received a nonsurgical relocation procedure without analgosedation. Only one patient (3.1\%) needed surgery with open eminectomy (Figure 2), due to previous surgical treatment of the TMJ.

Comorbidities were taken into consideration if they altered the anatomy of the TMJ or had an effect on the movements of the TMJ. Such comorbidities were present in only three patients $(9.4 \%)$, including previous eminectomy and ligamentorrhaphy, jaw ridge atrophy, as well as an isolated case of ligamentorrhaphy. In all three cases, the patients received conservative treatment (Table 1).

\section{Discussion}

Dislocation of TMJ is an infrequent but spectacular disease. The goal of treatment of any acute TMJ dislocation is to return the condyle to its original physiological and anatomical position. It is extremely important to take an accurate history and to analyze the exact etiology of the TMJ dislocation. Some interesting associations have been published, which make this apparently easy diagnosis more difficult. ${ }^{5}$ In one case, the first manifestation of the metastasis of a primary lung cancer was nonreducible dislocation of the mandible. ${ }^{20}$ Another case report describes an event of TMJ dislocation due to dystonia following a single dose of aripiprazole and another two cases of acute pure propranolol toxicity associated with bilateral TMJ dislocation. ${ }^{21,22}$

It is extremely important that the signs and symptoms are correctly diagnosed, so that treatment can be carried out as 
Table I Patient characteristics with temporomandibular joint dislocation

\begin{tabular}{|c|c|c|c|c|c|c|c|}
\hline $\begin{array}{l}\text { Patient ID } \\
\text { number }\end{array}$ & Sex & Age & Trigger & Side & Recurrence & Therapy & Comorbidities \\
\hline I & $M$ & 24 & Spontaneous & Right & Chronic recurrent & Conservative $^{a}$ & No \\
\hline 2 & $M$ & 24 & Spontaneous & Bilateral & Chronic recurrent & $\begin{array}{l}\text { Operation } \\
\text { (eminectomy) }\end{array}$ & No \\
\hline 3 & W & 43 & Spontaneous & Bilateral & Chronic recurrent & Conservative $^{b}$ & No \\
\hline 4 & $M$ & 25 & Spontaneous & Bilateral & Chronic recurrent & Conservative $^{b}$ & $\begin{array}{l}\text { Previous eminectomy and } \\
\text { ligamentorrhaphy }\end{array}$ \\
\hline 5 & W & 17 & Spontaneous & Left & Chronic recurrent & Conservative $^{a}$ & No \\
\hline 6 & $M$ & 35 & Spontaneous (while vomiting) & Left & Acute & Conservative $^{\mathrm{b}}$ & No \\
\hline 7 & W & 88 & Spontaneous & Left & Acute & Conservative $^{b}$ & Jaw ridge atrophy \\
\hline 8 & W & 28 & Spontaneous & Bilateral & Chronic recurrent & Conservative $^{\mathrm{a}}$ & No \\
\hline 9 & W & 87 & Spontaneous (during dental treatment) & Bilateral & Acute & Conservative $^{\mathrm{b}}$ & No \\
\hline 10 & W & 17 & Spontaneous & Bilateral & Chronic recurrent & Conservative $^{\mathrm{a}}$ & No \\
\hline II & $M$ & 85 & Spontaneous & Left & Chronic recurrent & Conservative $^{\mathrm{a}}$ & No \\
\hline 12 & W & 63 & Spontaneous & Left & Acute & Conservative $^{b}$ & No \\
\hline 13 & W & 31 & Spontaneous & Left & Acute & Conservative $^{a}$ & No \\
\hline 14 & $M$ & 34 & Spontaneous (while eating) & Right & Acute & Conservative $^{b}$ & No \\
\hline 15 & $M$ & 34 & Spontaneous (while eating) & Right & Chronic recurrent & Conservative $^{\mathrm{b}}$ & No \\
\hline 16 & $M$ & 27 & Spontaneous & Bilateral & Chronic recurrent & Conservative $^{b}$ & No \\
\hline 17 & $M$ & 63 & Spontaneous & Left & Chronic recurrent & Conservative $^{b}$ & No \\
\hline 18 & $M$ & 31 & Spontaneous & Bilateral & Chronic recurrent & Conservative $^{\mathrm{a}}$ & Previous ligamentorrhaphy \\
\hline 19 & W & 48 & Spontaneous (epileptic seizure) & Bilateral & Chronic recurrent & Conservative $^{b}$ & No \\
\hline 20 & M & 18 & Traumatic (fall) & Left & Acute & Conservative $^{\mathrm{b}}$ & No \\
\hline 21 & W & 75 & Spontaneous (during psychosis) & bilateral & Chronic recurrent & Conservative $^{\mathrm{b}}$ & No \\
\hline 22 & W & 92 & Spontaneous & Left & Acute & Conservative $^{b}$ & No \\
\hline 23 & W & 48 & Spontaneous (epileptic seizure) & Bilateral & Chronic recurrent & Conservative $^{b}$ & No \\
\hline 24 & M & 28 & Spontaneous & Bilateral & Chronic recurrent & Conservative $^{a}$ & No \\
\hline 25 & W & 22 & Spontaneous & Left & Chronic recurrent & Conservative $^{b}$ & No \\
\hline 26 & $M$ & 64 & Traumatic (fall) & Bilateral & Acute & Conservative $^{a}$ & No \\
\hline 27 & $M$ & 32 & Spontaneous & Bilateral & Acute & Conservative $^{\mathrm{a}}$ & No \\
\hline 28 & $M$ & 21 & Spontaneous & Bilateral & Chronic recurrent & Conservative $^{\mathrm{b}}$ & No \\
\hline 29 & W & 50 & Spontaneous & Bilateral & Chronic recurrent & Conservative $^{b}$ & No \\
\hline 30 & W & 27 & Spontaneous & Bilateral & Acute & Conservative $^{\mathrm{a}}$ & No \\
\hline 31 & W & 24 & Spontaneous & Bilateral & Acute & Conservative $^{\mathrm{a}}$ & No \\
\hline 32 & $M$ & 41 & Spontaneous & Bilateral & Chronic recurrent & Conservative $^{\mathrm{b}}$ & No \\
\hline
\end{tabular}

Notes: Conservative ${ }^{\mathrm{a}}$ : with analgosedation. Conservative ${ }^{\mathrm{b}}$ : without analgosedation.

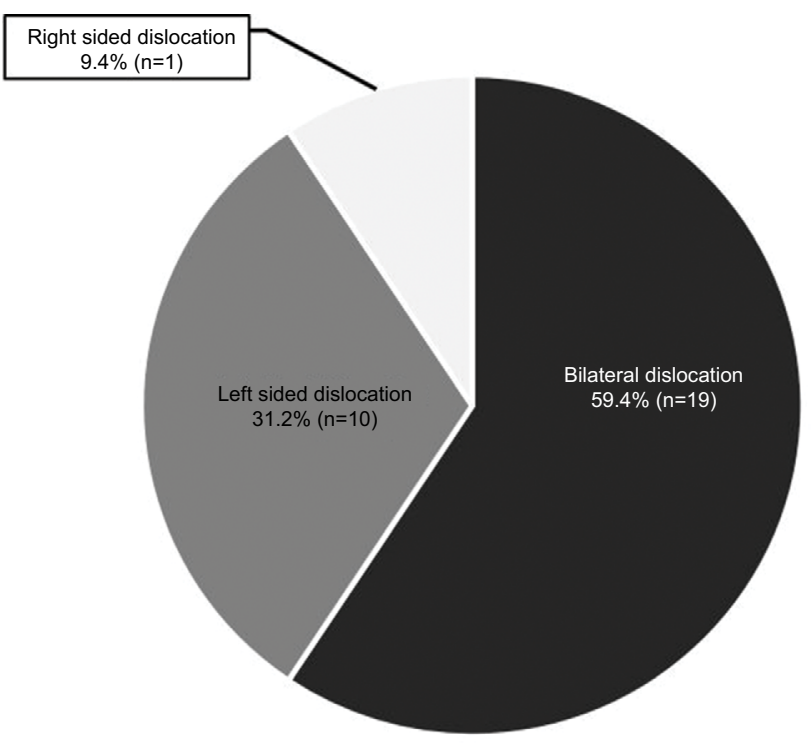

Figure I Percentage of temporomandibular joint dislocations related to the injured side. quickly as possible without further delay. ${ }^{7}$ The time between dislocation and relocation is absolutely crucial because once dislocation has taken place, spasms of the masseter and pterygoid muscles may worsen over time, causing the mandible to contract into the dislocated position, so that the reduction procedure becomes more difficult. If no treatment occurs for more than 2 weeks, fibrosis and even fractures become increasingly probable. ${ }^{9}$

The current retrospective descriptive study aims to make a global evaluation of TMJ dislocations in the ED of an acute level I trauma center in Switzerland. The Department of Emergency Medicine, Inselspital, University Hospital Bern, University of Bern, is the only level 1 center in Canton Bern, serves about 1.8 million people and treats more than 46,000 adult patients per year (2016). This is one of the first studies that systematically analyzes the characteristics of the patients involved in an accident or a spontaneous event with TMJ 


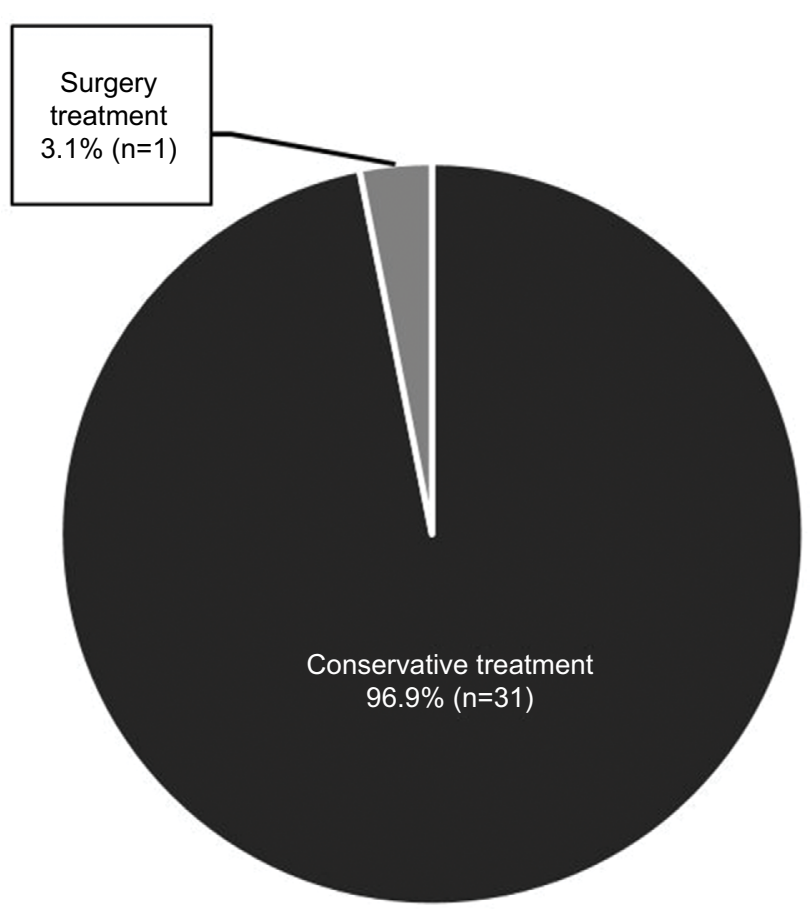

Figure $\mathbf{2}$ Therapy choice during temporomandibular joint dislocation.

dislocation in Switzerland, and there have been few reports from other countries. Other studies have focused on relocation procedures during TMJ dislocation ${ }^{13}$ or have proposed new techniques. ${ }^{23-26} \mathrm{We}$ aimed to analyze systematically the patients who presented with TMJ dislocation in our ED, their characteristics, and the treatment methods that they had received.

Our results showed balanced gender distribution, with a median age of 42.06 years. The balanced gender distribution is in contrast with the international literature, in which female predominance is reported. ${ }^{5,23}$ On the contrary, the median age seems to be the same as in other studies. ${ }^{23}$

Bilateral and acute TMJ dislocation was most common (59.4\%). Unfortunately, there are not enough data available, which enable us to make a comparison.

In our study on 32 patients, 20 (62.5\%) had a previous history of TMJ dislocation. A previous study reported that of the 31 patients, only nine (29\%) had previously suffered TMJ dislocation. ${ }^{23}$ However, they only analyzed acute, nontraumatic patients with TMJ dislocation and as a result, no comparison can be performed.

In addition, our findings reported a marked incidence (93.7\%) of nontraumatic etiology in TMJ dislocation as well as the predominant choice of the conservative treatment method (with or without analgosedation), which is consistent with many other studies. ${ }^{7,13,23}$ Both cases $(6.3 \%)$ of traumatic etiology in our study were due to falls. This etiopathogenesis is widely known; 5 however, it is not the only one. In the nontraumatic causes, we found single episodes of TMJ dislocation during dental treatment, during psychosis, two cases while eating, and two others while vomiting. Some of these reasons had been reported, ${ }^{23}$ but the limited number does not enable us to make a comparison. We noted that of the patients who received conservative treatment, 19 (61.3\%) had successful TMJ replacement without analgosedation. No comparable data have been published. The results suggest that the patients were rapidly treated. However, the time periods from the dislocation of the TMJ till the presentation in the ED and till the moment in which a successful TMJ replacement was performed were not measured. It would also not be ethically justifiable to delay treatment in some patients to examine whether the time period until TMJ replacement has an effect on the success rate. Finally, TMJ replacement can be performed by emergency staff. For educative purposes, a quick view of the reposition technique is recommended for the emergency surgeon shortly before reposition. ${ }^{27}$ No complication due to the reposition technique was observed in our retrospective analysis.

\section{Limitations}

Our findings have to be considered with some caution, as the study was conducted retrospectively. As the information in our medical history database is presented in a narrative comment, there is no guarantee that the number of patients with TMJ dislocation was fully reported. Furthermore, our study was limited to adults ( $\geq 16$ years old), as children are treated at a separate ED in our hospital. Because the direction of the dislocation (anterior, posterior, superior, or lateral) in each patient was not reported, as a first approximation, we analyzed our data by uni- (right or left) or bilateral TMJ dislocation. Moreover, many studies have focused on analyzing or proposing new relocation methods. ${ }^{13,23-26}$ Unfortunately, our registered data did not document the relocation method selected for each patient.

\section{Conclusion}

TMJ dislocation is not a frequent condition. When it occurs, accurate diagnosis and rapid treatment are needed.

Both acute hospitals and private dental practices should be familiar with targeted diagnosis and effective relocation. Manual reduction is sufficient in case of acute dislocation. In cases of repetitive dislocations or complicated situations, surgery is recommended.

\section{Disclosure}

The authors report no conflicts of interest in this work. 


\section{References}

1. Sharma NK, Singh AK, Pandey A, Verma V, Singh S. Temporomandibular joint dislocation. Natl J Maxillofac Surg. 2015;6(1):16-20.

2. Snell SN. Clinical Anatomy by Regions. 8th ed. Baltimore (MD): Lippincott Williams and Wilkins, 2008.

3. Candirli C, Yüce S, Cavus UY, Akin K, Cakir B. Autologous blood injection to the temporomandibular joint: magnetic resonance imaging findings. Imaging Sci Dent. 2012;42(1):13-18.

4. Mcgoldrick DM, Stassen LF. Management of acute dislocation of the temporomandibular joint in dental practice. J Ir Dent Assoc. 2010;56(6):268-270.

5. El Bouazzaoui A, Labib S, Derkaoui A, Adnane Berdai M, Bendadi A, Harandou M. Dislocation of temporo-mandibular joint - an uncommon circumstance of occurrence: vaginal delivery. Pan Afr Med J. 2010; 5:23.

6. Sailors ME. Evaluation of sports-related temporomandibular dysfunctions. J Athl Train. 1996;31(4):346-350.

7. Girish K, Syed S, Shashi S.C, Khan M. Management of temporomandibular joint dislocation: review of literature. Int J Sci Res. 2016;5(1):574-577.

8. Liddell A, Perez DE. Temporomandibular joint dislocation. Oral Maxillofac Surg Clin North Am. 2015;27(1):125-136.

9. Pillai S, Konia MR. Unrecognized bilateral temporomandibular joint dislocation after general anesthesia with a delay in diagnosis and management: a case report. J Med Case Rep. 2013;7:243.

10. Oliphant R, Key B, Dawson C, Chung D. Bilateral temporomandibular joint dislocation following pulmonary function testing: a case report and review of closed reduction techniques. Emerg Med J. 2008;25(7):435-436.

11. Tauro D, Lakshmi S, Mishra M. Superolateral dislocation of the mandibular condyle: report of a case with review of literature and a proposed modification in the classification. Craniomaxillofac Trauma Reconstr. 2010;3(3):119-123.

12. Thangarajah T, McCulloch N, Thangarajah S, Stocker J. Bilateral temporomandibular joint dislocation in a 29 -year-old man: a case report. J Med Case Rep. 2010;4:263.

13. White T, Hedderick V, Ramponi DR. Dislocation of the temporomandibular joint and relocation procedures. Adv Emerg Nurs J. 2016;38(3):177-182.

14. Adekeye EO, Shamia RI, Cove P. Inverted L-shaped ramus osteotomy for prolonged bilateral dislocation of the temporomandibular joint. Oral Surg Oral Med Oral Pathol. 1976;41(5):568-577.
15. Srivastava R, Jyoti B, Devi P. Oral splint for temporomandibular joint disorders with revolutionary fluid system. Dent Res J (Isfahan). 2013;10(3):307-313

16. Wright EF, North SL. Management and treatment of temporomandibular disorders: a clinical perspective. J Man Manip Ther. 2009; 17(4):247-254

17. Hoglund LT, Scott BW. Automobilization intervention and exercise for temporomandibular joint open lock. J Man Manip Ther. 2012;20(4):182-191.

18. Akinbami BO. Evaluation of the mechanism and principles of management of temporomandibular joint dislocation. Systematic review of literature and a proposed new classification of temporomandibular joint dislocation. Head Face Med. 2011;7:10.

19. Sato J, Segami N, Nishimura M, Suzuki T, Kaneyama K, Fujimura K. Clinical evaluation of arthroscopic eminoplasty for habitual dislocation of the temporomandibular joint: comparative study with conventional open eminectomy. Oral Surg Oral Med Oral Pathol Oral Radiol Endod. 2003;95(4):390-395.

20. Boniello R, Gasparini G, D'Amato G, Di Petrillo A, Pelo S. TMJ metastasis: A unusual case report. Head Face Med. 2008;4:8.

21. Solomon S, Gupta S, Jesudasan J. Temporomandibular dislocation due to aripiprazole induced dystonia. Br J Clin Pharmacol. 2010;70(6):914-915.

22. Aghabiklooei A, Elahi H, Mostafazadeh B. Temporomandibular joint dislocation due to acute propranolol intoxication. Int Med Case Rep J. 2010;3:59-61.

23. Gorchynski J, Karabidian E, Sanchez M. The "syringe" technique: a hands-free approach for the reduction of acute nontraumatic temporomandibular dislocations in the emergency department. J Emerg Med. 2014;47(6):676-681.

24. Hasson O, Nahlieli O. Autologous blood injection for treatment of recurrent temporomandibular joint dislocation. Oral Surg Oral Med Oral Pathol Oral Radiol Endod. 2001;92(4):390-393.

25. Ardehali MM, Kouhi A, Meighani A, Rad FM, Emami H. Temporomandibular joint dislocation reduction technique: a new external method vs. the traditional. Ann Plast Surg. 2009;63(2):176-178.

26. Heidari SF. The new technique for reduction of bilateral mandibular dislocation. Am J Emerg Med. 2015;33(9):1327.e3.

27. Zweifel DF, Pietramaggiori G, Broome M. Videos in clinical medicine. Repositioning dislocated temporomandibular joints. $N$ Engl $J$ Med. 2014;370(6):e9.

28. Yasuoka T, Nakashima M, Okuda T, Tatematsu N. Effect of estrogen replacement on temporomandibular joint remodeling in ovariectomized rats. J Oral Maxillofac Surg. 2000;58(2):189-196; discussion 196-197.
Open Access Emergency Medicine

\section{Publish your work in this journal}

The Open Access Emergency Medicine is an international, peerreviewed, open access journal publishing original research, reports, editorials, reviews and commentaries on all aspects of emergency medicine. The manuscript management system is completely online and includes a very quick and fair peer-review system, which is all

\section{Dovepress}

easy to use. Visit http://www.dovepress.com/testimonials.php to read real quotes from published authors. 